\title{
A dictionary of visual analogies
}

\author{
Luciano Perondi ${ }^{1}$, Marco Ferrari ${ }^{2}$ and Donato Ricci ${ }^{3}$ \\ 1,2 \\ ISIA Urbino, via Santa Chiara 36, 36100 Urbino, Italy \\ \{luciano.perondi, marco.ferrari\}@isiaurbino.net \\ ${ }^{3}$ SciencesPo/médialab, Paris, France \\ donato.ricci@sciencespo.fr
}

\begin{abstract}
This paper discusses a theoretical framework for a research aimed to produce a dictionary of visual analogies used for the explanation of scientific theories, collected both from historical and contemporary sources. The artifacts will be indexed through a set of criteria and tags that will allow to navigate the contents and map correlations across time, scientific domains and types of publication. The archive will grow as an open-ended accumulation of examples, adapting the methodology for the selection and organisation of the analogies based on the new entries. A set of visualisations will be used in order to navigate the archive and make emerging patterns legible. The initial method of classification will be based on the faceted system envisioned by Luca Rosati (Rosati, 2015), in which artifacts are tagged and tags are organised according to a faceted classification. Tags will not be mutually exclusive, but they'll act like attributes: each entry may have multiple tags, the number of which can grow without any limit or predetermined direction.
\end{abstract}

Keywords: Analogy / Information Design / Scientific Representation

\section{A Definition of Analogy}

In order to understand if analogies can be considered a valuable tool to provide legibility into complex domains of information, we need first to define what kind of analogies we consider in our enquiry. As an initial reference, we introduce the discrimination between two different definitions of analogy, as proposed by Gelfert (2016):

"The term 'analogy', which derives from the Greek expression for 'proportion', itself has multiple uses, depending on whether one considers its use as a rhetorical device or as a tool for scientific understanding."

Given these two intents, the one that is of interest here is the second one, and specifically the one that defines analogy as the resemblance between relations in two different domains: the relation $(a-b)$ in domain-1 is like the relation $(c-d)$ in domain2 (Bailer-Jones, 2002; Gelfert 2016). 
"Its general form is that of 'pointing to a resemblance between relations in two different domains, i.e. $A$ is related to $B$ like $C$ is related to $D^{\prime \prime \prime}$ (Bailer-Jones, 2002, p. 110).

According to Pierre Duhem, who criticised the excessive use of mechanical models for the visualisation of physical processes in scientific literature:

"Analogies consist in bringing together two abstract systems; either one of them already known serves to help us guess the form of the other not yet known, or both being formulated, they clarify the other." (Duhem, 1914/1954)

We can visually express the inference at the basis of this particular type of analogy with the sketch of fig. 1 :

\section{domain-1 domain-2}

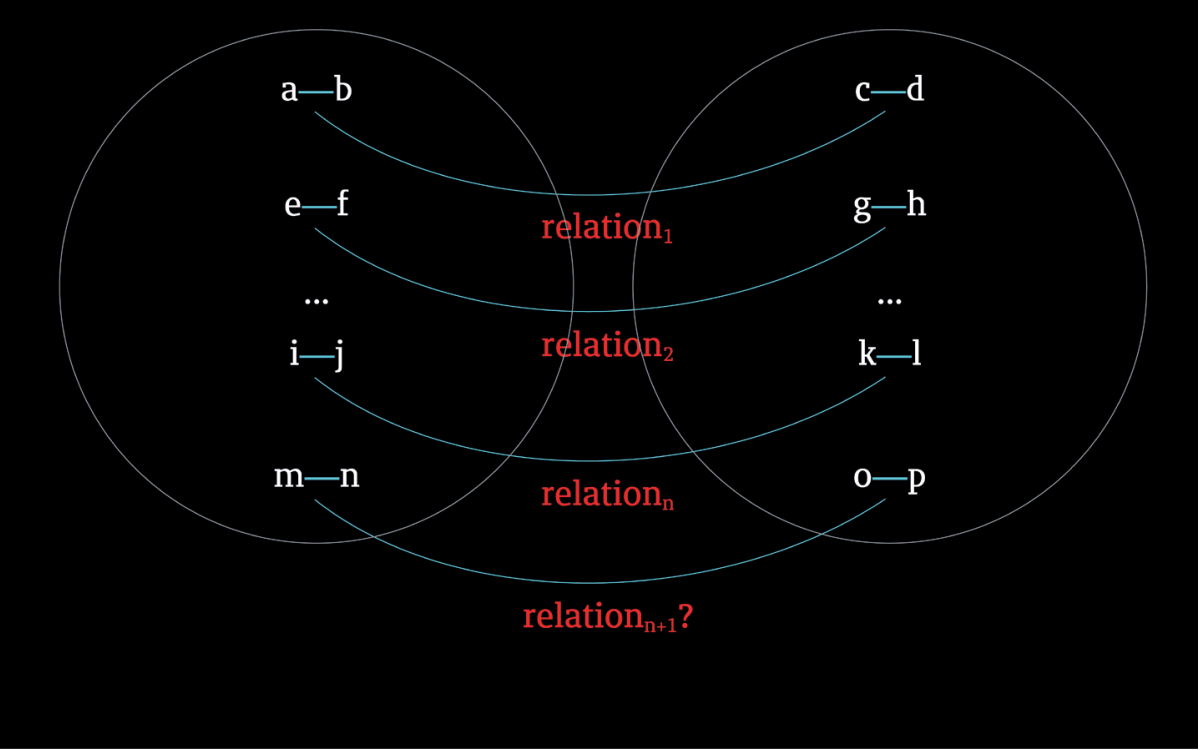

Fig. 1. If $\mathrm{n}$ properties in domain-1 match with the ones in domain-2, we can conclude that also the property^ $n+1$ institutes a valid relation between the two domains.

In real-life examples, it's a common situation that just some of all of the possible relations are used to establish a valid analogy, and we don't know if there is a not- 
matching relation until we find it. Even in the case of a non-complete correspondence between two different domains, though, this condition does not jeopardise the legitimacy of analogy as a tool for understanding. Analogies do not necessarily imply structural identity, but help to find patterns and similarities between two apparently distant domains.

\section{domain-1 domain-2}

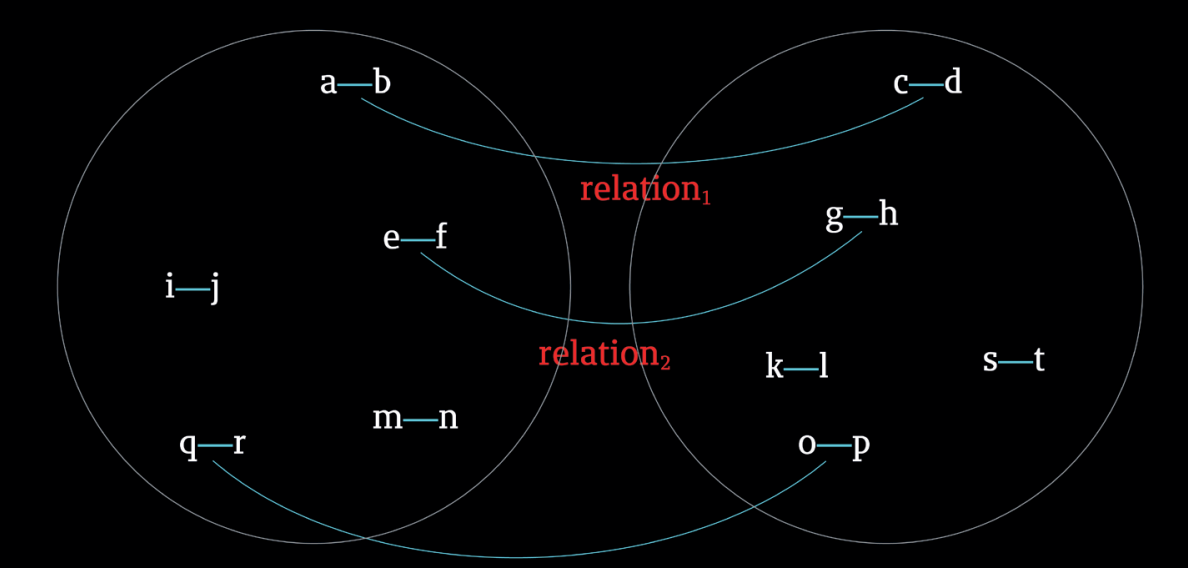

relation $_{3}$

Fig. 2. Despite the apparent limitation of a partial correspondence between features in two different domains, analogies can be used anyway as an effective tool for understanding: finding relations that do not match is also a knowledge-building process. Non-matching relationships can also be negative analogies.

It's interesting to note that analogies are often created between non-compatible domains. This means that inference per se is not enough to explain the process through which an analogy is established. Analogies-like scientific discoveries-are the product of creative imagination (Hesse, 1953), and are actively constructed.

As an example, let's consider the analogy used by Christiaan Huygens to explain the behaviour of sound waves and light waves... (Gelfert, 2016). 
Another, well-known example is the analogy between the Bohr model of the atom and a planetary system. This analogy constitutes a hybrid comparison, since the behaviour of electrons that jump continuously between different orbits cannot be explained by the Saturnian model initially adopted by Rutherford. On the other hand, the fact that the electrons revolve around the central core is explained through the mechanical model of the solar system (Arabatzis and loannidou, 2015). Therefore, the evolution of the Borh model has been based both on a positive and a negative analogy: the contradictory behaviour of the electrons compared to the model allowed to find an alternative theory.

Both these analogies include incongrous features, but they've been fundamental for the study of their corresponding phaenomena.

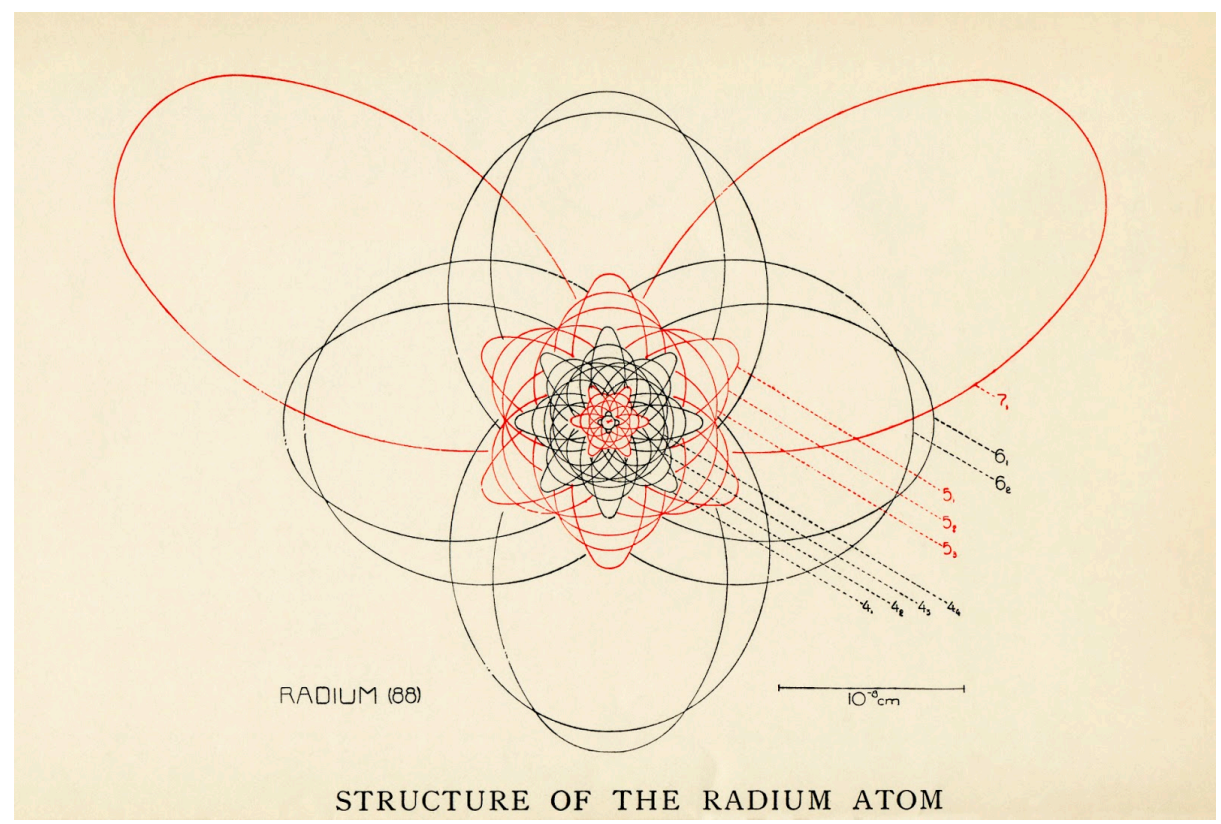

Fig. 3. The structure of the Radium atom according to Bohr's theory. Image from The atom and the Bohr theory of its structure. An elementary presentation, by Hendrik Anthony Kramers and Helge Holst (1923).

The use of analogy as a tool for the production of knowledge is not limited to the scientific domain, but it's common in many other fields. Let's consider as an example the kennings for Old Norse poetry, or the late Middle Age preaching based on an analogic process of building images (Bolzoni, 2002). We suppose that analogy is structural in human thinking, and thus it can be found in amy different fields as a way to articulate complex thoughts or elaborate knowledge. 
Metaphors share the same assumptions that we introduced for the definition of analogy, which means that they need to satisfy the following requisite: "a similarity between two different domains, where the features of domain-1 are structured as one-to-one relationships with the ones in domain-2".

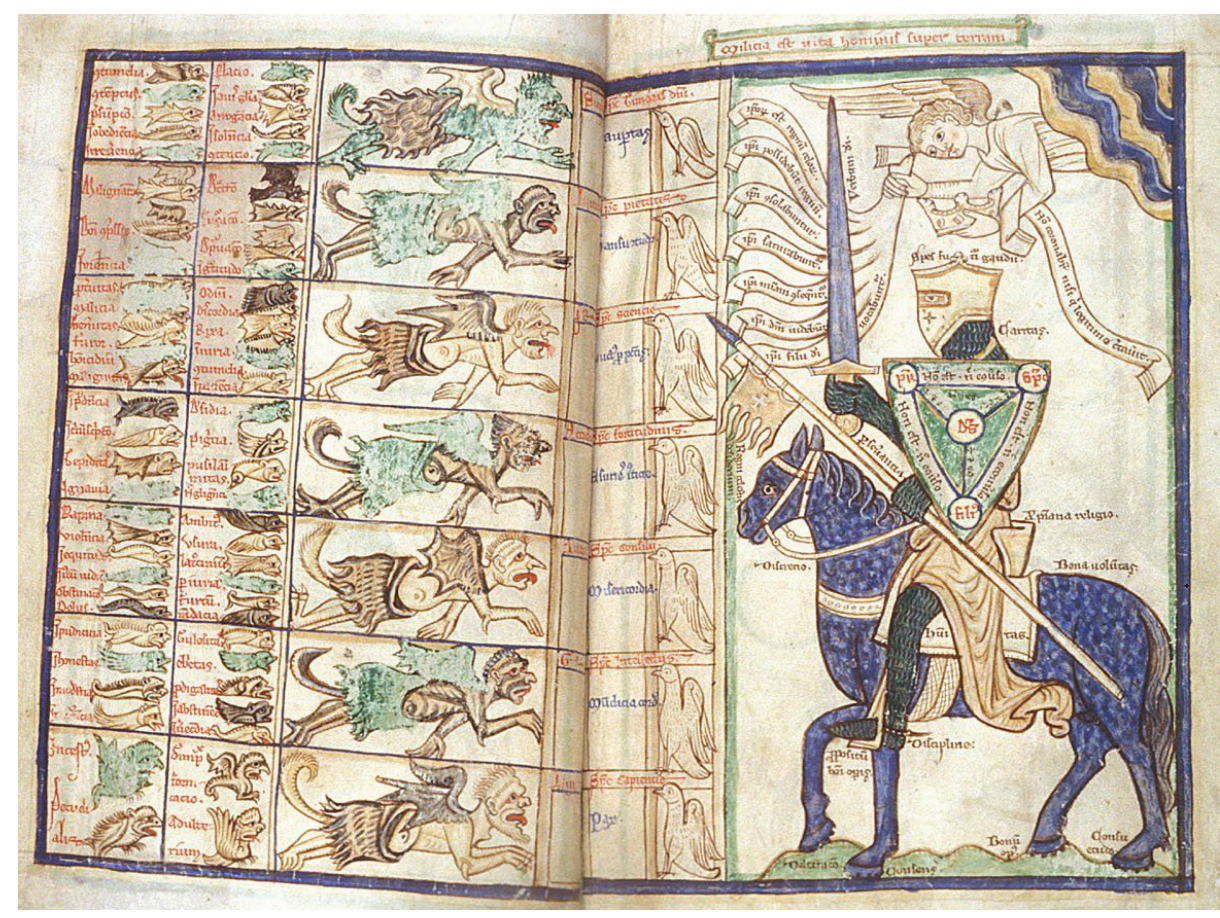

Fig. 4. Anonymous, Guilelmus Peraldus, Summa de virtutibus et vitiis, second half of XII century. Pigments on vellum. Harleian ms. 3244, folios 27v-28r.

\section{Structural Knowledge}

The second concept that we use to build our own model of analogy is the one of 'structural knowledge' as proposed by Jonassen (2000) in the field of problem-solving theories:

Analogies allow to make the first step to get a structural knowledge of a domain. According to Jonassen: "Structural knowledge [...] connects declarative and procedural knowledge. Structural knowledge is knowledge of how the ideas within a domain are integrated and interrelated" (Jonassen, 2000). 
The gap between declarative and procedural knowledge is bridged by analogies.

Structural knowledge is the knowledge of the relations within a domain, while analogy encompasses the similarities between relations within two different domains.

If we consider all the above points, referring to the definition of analogy and the concept of structural knowledge, we can state that analogies are a legitimate tool for accessing knowledge, and have been widely used over history to assemble scientific knowledge. C

We consider a definition of analogy in the widest possible sense: one that includes any kind of resemblance or likeness between domains. As stated before, the fundamental requisite is a correspondence or a partial similarity, for the purpose of explanation or clarification.

The reasons behind our interests in visual analogies are:

1. Analogies have always been part of the human understanding both of the physical world and abstract thought, but they are increasingly relevant and widespread today, where the visibility of technologies, systems and processes is being highly reduced by their exponential complexity and miniaturisation.

2. We want to trace an evolutionary history of analogies, and map how similar images have been used over time to define different concepts. The life of analogies often exceeds the one of the theories they represent and explain, in order to influence the subsequent evolution of scientific theories.

\section{A Charted History of Analogies}

The primary field of investigation will be limited to static and graphic representations used in scientific manuals published between the XIX and XX century. The analysis will be pointed only to visual and graphic artifacts (illustrations, diagrams, photos and photographic collages used for the explanation and divulgation of scientific theories), in order to limit the complexity of the methodological approach. The visual domain is the one we know better, and it will allow for a quicker processing of the images through visual analysis. The images will be sourced from publications present in the Internet Archive, a "non-profit, digital library of Internet sites and other cultural artifacts in digital form." (https://archive.org/about/). The Internet Archive provides free online access to a wide selection of manuals and publications from different scientific domains. In particular, the initial selection of images will be focused on scientific manuals. 
An initial corpus of images will be assembled in order to test the classification methodology and build a wide-enough database of contents that will then serve as a basis and reference for next step of the project, where the users of the dictionary will be able to suggest new entries. The aim is to build an open platform for the collection and classification of the images, an expanding database of visual analogies that will help to visualise the evolution of scientific theories throughout history.

Once this metholodgy will be tested and expanded with a suitable set of tags, it will then potentially be applied to other types of publication: encyclopedias, scientific papers, atlases, school textbooks, etc. We decided to initially focus on the manual because manuals have been the primary tool for the dissemination and affirmation of scientific theories throughout the XIX and XX century within peers. Other, more divulgative texts would weaken the analogies and their power to embody and steer scientific discoveries, as they would be seen just a mean for the spread of top-down knowledge.

Visual analogies found in manuals will be compared to similar ones in the same publication, besides looking for similar graphic artifacts outside the perimeter of the domain (snowball sampling).

The aim is to develop a web platform where the collection of the visual samples that constitute the database will be sorted and displayed, according to the following organisation. This tool that will serve as a way to cross-reference and investigate different analogies across time and disciplines, helping to map their relevance, cultural relevance, impact on the scientific discourse and mutual influence.

The initial method of classification will be based on the faceted system envisioned by Luca Rosati (Rosati, 2015), in which artifacts are tagged and tags are organised according to a faceted classification. Tags will not be mutually exclusive, but they'll act like attributes: each entry may have multiple tags, the number of which can grow without any limit or predetermined direction. We will thus be able to maintain the advantages of the tag classification-namely the possibility to introduce more attributes for each example and to introduce new attributes at every given momentplus the advantages of the faceted classification, used for the organisation of the attributes. 


\section{References}

Arabatzis, T., \& loannidou, D. (2015). The role of models and analogies in the Bohr atom. In F. Aaserud and H. Kragh (eds.), One Hundred Years of the Bohr Atom (pp. 360-376). Copenhagen: The Royal Danish Academy of Sciences and Letters.

D. Bailer-Jones, Models, Metaphors and Analogies, in The Blackwell Guide to the Philosophy of Science, ed. by P. Machamer, M. Silberstein (Blackwell, Oxford, 2002), pp. 108-127

Bolzoni, L. (2009). La rete delle immagini: predicazione in volgare dalle origini a Bernardino da Siena. Piccola biblioteca Einaudi. Einaudi. Retrieved from:

https://books.google.it/books?id=sHVnQAAACAAJ

Duhem, P. M. M., Wiener, P. P., Vuillemin, J., \& de Broglie, L. (1991). The Aim and Structure of Physical Theory. Atheneum paperbacks. Princeton University Press. Retrieved from https://books.google.it/books?id=5mVPK7QBdTkC

Gelfert, A. (2015). How to Do Science with Models: A Philosophical Primer. SpringerBriefs in Philosophy. Springer International Publishing. Retrieved from:

https://books.google.it/books?id=KVFECWAAQBAJ

Hesse, M. (1953). Models in Physics. The British Journal for the Philosophy of Science, 4(15), 198-214. Retrieved from http://www.jstor.org/stable/685897

Jonassen, D. H. (2000). Computers as Mindtools for Schools: Engaging Critical Thinking. Merrill. Retrieved from https://books.google.it/books?id=IW3uAAAAMAAJ

Kramers, H. A. (2015). The Atom and the Bohr Theory of Its Structure, an Elementary Presentation. Fb\\&c Limited. Retrieved from:

https://books.google.it/books?id=oXKltAEACAAJ

Rosati, L. (2015). Combinare tag e classificazione a faccette per migliorare la trovabilità. Retrieved from https://www.lucarosati.it/blog/tag-e-faccette 